\title{
Écologie de l'institution scolaire - La cohérence entre contenus, méthodes et milieu scolaire
}

\section{Mario Salomone}

\section{(2) OpenEdition \\ Journals}

Édition électronique

URL : http://journals.openedition.org/ere/3899

DOI : $10.4000 /$ ere.3899

ISSN : 2561-2271

Éditeur

Centr'ERE

Référence électronique

Mario Salomone, «Écologie de l'institution scolaire - La cohérence entre contenus, méthodes et milieu scolaire », Éducation relative à l'environnement [En ligne], Volume 6 | 2007, mis en ligne le 14 septembre 2007, consulté le 21 février 2020. URL : http://journals.openedition.org/ere/3899 ; DOI : 10.4000/ere.3899 


\title{
Écologie de l'institution scolaire - La cohérence entre contenus, méthodes et milieu scolaire
}

\author{
Mario Salomone
}

1 L'éducation relative à l'environnement (ERE) ne peut donner une contribution décisive à la transformation des institutions scolaires que si elle relève le défi d'en couvrir simultanément tous les aspects, à savoir: mission fondamentale; contenus et programmes; relations de partenariat avec les autres institutions et les composantes de la société civile ; expérimentation et réinvestissement des concepts appris au niveau du territoire; protagonisme social de l'institution scolaire; méthodes didactiques; utilisation des nouvelles technologies de l'information et de la communication; organisation et gestion du milieu d'apprentissage (considéré aussi bien au sens strict espaces physiques où se déroulent des activités d'apprentissage structurées -, qu'au sens large du terme), voire les lignes directrices qui réglementent la conception architecturale, les normes de sécurité et les standards pour le bien-être physique des usagers des bâtiments abritant les écoles ou les universités.

2 En règle générale, ces aspects sont affrontés séparément et ils sont dans la plupart des cas confiés à des rôles socioprofessionnels et à des domaines disciplinaires différents : les politiciens, les pédagogues, les professeurs des différentes disciplines, les dirigeants scolaires, les psychologues, les sociologues, les experts de processus organisationnels et de systèmes de qualité, les administrateurs publics, etc. La raison de cette nécessité d'intégration et de cohérence entre tous les aspects réside dans les principes sur lesquels l'ERE se base et qui sont liés à l'idée de complexité et de multiplicité présente aussi bien dans l'humanité mondialisée que dans la vision cosmologique.

3 Sans s'attarder sur une réflexion désormais ressassée depuis plusieurs dizaines d'années sur la portée épistémologique et éthique de l'éducation à l'environnement, il convient toutefois de rappeler que pour éduquer le citoyen, l'école et l'université doivent « expliciter et affronter sa condition évolutive et planétaire » (Bocchi et Ceruti, 2004, p. 53). L'évolution humaine est celle de la planétarisation et de la multiculturalité. 
La vision du monde est graduellement passée du géocentrisme à l'idée d'univers multiples (« notre espace, notre temps, notre univers même, ne sont peut-être que des fragments d'un plurivers incomparablement plus vaste et plus diversifié », Bocchi et Ceruti, 2004, p. 196), la stupeur s'est accrue et la confiance dans des vérités absolues ou dans la possibilité de décrire l'univers avec quelques lois fondamentales s'est effondrée. Alors, si les jeux ne sont pas encore faits, si le futur n'est pas certain, si l'avenir doit être vivable, nous sommes invités à considérer le monde comme « une construction à laquelle nous pouvons tous participer» (Prigogine, 1999). L'histoire est une succession de bifurcations, elle est faite de fluctuations à l'échelle microscopique desquelles dépendent les bifurcations que les événements prendront. Et les fluctuations sont le résultat d'actions individuelles.

4 Bref, nous pouvons appeler « éducation relative à l'environnement » une éducation qui offre les outils pour construire un monde possible, c'est-à-dire qui propose les éléments de base de la pensée complexe et qui introduit des fondements éthiques : éthique de la responsabilité, de l'équité, de la solidarité, de la précaution, du respect de l'altérité, des différences et des droits des minorités. En outre, l'ERE embrasse le vaste domaine des connaissances théoriques, techniques et organisationnelles nécessaires aux nouveaux processus de bioéconomie, les capacités cognitives et relationnelles requises par le fait de devoir se confronter avec un ensemble de disciplines et avec une multiplicité d'autres sujets, la familiarité avec les formes de nouvelle gouvernance et de conception participée et avec les méthodologies de négociation des conflits.

\section{Écologie à tous les niveaux}

Or, ce programme de réforme de l'éducation ne peut être réalisé sans un profond changement du rapport entre l'école et la société, sans un profond changement des techniques didactiques et sans une écologie des bâtiments, une réduction des émissions, une pratique de styles de vie et de comportements écologiques, une écologie de l'organisation des temps, une écologie de la communication interne, une écologie de l'utilisation des structures. La motivation d'un parcours de cohérence étendu, profond, constant et convaincu est évidente : en effet, d'une part on ne peut pas transmettre par les comportements un message discordant par rapport à la mission éducative ; d'autre part la situation vécue devient aussi bien une occasion de laboratoire et d'apprentissage sur le terrain (pour comprendre, en les pratiquant, que les solutions existent et que la durabilité est réalisable si l'on en a la volonté et la fantaisie) qu'une contribution réelle à l'amélioration de la situation globale, un exemple que la durabilité est faite de mille choix concrets et de gestes quotidiens.

6 D’après David Orr (1994), par exemple, l'éducation doit être reconstituée d'après six principes :

1. toute éducation est une éducation à l'environnement ;

2. l'objectif de l'éducation n'est pas de maîtriser le contenu, mais soi-même;

3. la connaissance implique la responsabilité de son bon usage ;

4. nous pouvons affirmer que nous connaissons quelque chose seulement quand nous en comprenons les effets sur les personnes et les communautés ;

5. l'importance des détails, la force des exemples par rapport aux mots, la cohérence entre le mot et l'action ;

6. les modalités d'apprentissage sont tout aussi importantes que les contenus. 
7 Donc, les lieux où se déroulent les activités doivent être le premier champ d'application des solutions techniques et organisationnelles pour la durabilité, en respectant des paramètres aussi bien physico-chimiques que relationnels, en réduisant les consommations et en augmentant la communication, tandis que le territoire doit devenir le terrain d'une prise en charge de l'environnement et d'actions positives de transformation.

Les écoles et les universités doivent profondément changer et rénover, dans l'optique de l'éco-développement, le système des études, leur organisation et leurs méthodes. [...] l'éducation ne changera pas réellement si les institutions éducationnelles ne deviennent pas elles-mêmes « durables » et cohérentes avec les principes de l'éco-développement et si la didactique, qui devra s'ouvrir à la recherche-action et au rapport avec la communauté, ne change pas. (WEEC, 2005).

8 Bref, la conversion de la perspective culturelle et l'orientation éthique à la responsabilité vers le monde ne peuvent évidemment pas rester sur un plan purement théorique, d'adhésion idéale et pour ainsi dire « cérébrale ». Et ce pour toute une série de bonnes raisons :

1. L'institution scolaire du passé transmettait les valeurs dominantes : à travers l'éducation, la société s'autoperpétuait. Ceci explique, sans toutefois la justifier, la séparation d'antan. Comme l'observe, par exemple, François Dubet (2002), jusqu'aux années 70 du XXe siècle, l'école était un système fortement hiérarchisé, mais qui réussissait en quelque sorte à neutraliser ses contradictions dans la mesure où il se présentait comme un sanctuaire fondé sur des principes sacrés et homogènes et dans lequel les enseignants entraient par vocation. Pendant ces deux derniers siècles et notamment ces derniers temps, la pédagogie et l'innovation didactique ont essayé de détourner l'école de son caractère idéologique et de dépasser la simple transmission de notions. Aujourd'hui, on demande à l'éducation de dépasser définitivement la séparation et de s'intégrer plus strictement dans le tissu social. L'extension tous azimuts du choix de l'éducation relative à l'environnement comme principe-guide d'une réforme éducationnelle peut indubitablement contribuer à accomplir la transformation de la fonction de l'institution scolaire survenue pendant ces dernières décennies.

2. Concevoir l'éducation non pas comme une simple transmission du savoir ou comme une formation aux différents rôles sociaux, mais comme un outil de transformation implique un devoir de témoignage, un appel à l'engagement : ce qui est enseigné doit être confronté au monde réel où, du reste, les apprenants sont plongés. Aujourd'hui, les outils de citoyenneté sont essentiellement fournis par l'éducation, c'est-à-dire par la capacité de comprendre et de maîtriser les grandes questions du monde contemporain, sans laquelle, par exemple, il n'est pas possible de se faire une opinion sur les sujets abordés ou d'exprimer un jugement conscient. L'apprentissage doit donc aller au-delà de l'étroit horizon personnel et de l'« espace » limité pour nous faire prendre conscience des interconnexions globales.

3. Il existe une responsabilité sociale de la connaissance, une responsabilité cognitive: l'utilisation du savoir comporte une grande responsabilité sociale, dans la recherche et dans l'évaluation des données, dans le scrupule et dans le degré d'approfondissement des hypothèses de recherche, dans le choix du schéma mental à suivre, dans les valeurs qui guident la définition des hiérarchies de priorité et les décisions, petites ou grandes qu'elles soient, etc., dans la mesure où les études de faisabilité, les avis, les projets, les stratégies, les solutions techniques se transforment en une réalité concrète, voire non modifiable et irréversible. Cette responsabilité ne concerne pas seulement la science (qui a notoirement «perdu son innocence » au moment où explosait la Bombe sur Hiroshima), mais aussi tout cadre, tout technicien, tout décideur, et plus généralement toute personne, et elle doit être 
immédiatement mise en pratique : sa vérification concrète ne doit pas être repoussée à l'issue du parcours scolaire et à l'entrée dans la vie active.

\section{Lien avec l'environnement}

1. Par rapport à une société rigidement divisée en groupes et en classes (parmi lesquelles l'institution scolaire peut favoriser une mobilité ascensionnelle) et organisée en corps fermés et en institutions séparées, la société actuelle, mobile, interconnectée, multiculturelle et multiethnique fait de plus en plus émerger une structure réticulaire qui la rend plus semblable à la vie de la planète dont la toile peut être considérée comme un schéma général. La recherche sociologique et la recherche anthropologique mettent de plus en plus l'accent sur l'individu et sur ses relations par rapport au groupe organisé, institutionnalisé, c'est-à-dire constitué d'une structure comme l'école, l'église, l'entreprise, l'administration locale, le gouvernement, le syndicat (Piselli, 2001).

L'idée de réseau nous conduit forcément à un social constitué des relations qui se créent entre les personnes, entre les personnes et les éléments matériels et symboliques de l'environnement et plus largement entre les groupes, les organisations, les institutions. (Amerio et Croce, 2000, p. 333)

9 Ceci semble encore plus vrai dans la société de l'ère de la mondialisation et de ce grand réseau qu'est la toile mondiale de l'Internet. La communauté se casse en morceaux qui développent des relations (de négociation, coopératives, institutionnelles) à différentes échelles spatiales. L'activité économique elle-même est enchâssée dans les rapports sociaux et influencée par ces derniers, mais l'« autre économie » aussi est caractérisée par une structure de réseau. Il suffit de penser aux réseaux d'économie solidaire, à l'économie du don, au troc, aux banques du temps (par exemple: Coluccia, 2001 et 2002) : le réseau est un élément constitutif de l'économie écologique, celle-ci exigeant une approche endogène et donc attentive au territoire en tant qu'espace réel, concret, historique de l'action humaine. Mais c'est en même temps une approche par le bas et donc fondée sur la dimension relationnelle, c'est aussi un développement local et donc de toute une communauté organisée en réseau (par exemple : Becattini et Sforzi, 2002; Sacco et Zamagni, 2002; Grasselli, 2003). Par conséquent, les réseaux sont l'une des façons les plus cohérentes de concrétiser l'action environnementale: les réseaux de personnes, de sujets publics et privés et de services deviennent une façon, peut-être même la façon la plus adéquate aux mécanismes profonds des rapports sociaux, de construire un langage commun, d'assurer des formes coopératives d'intervention sur la réalité, de se coordonner, de s'échanger de bonnes pratiques.

Dans ce contexte, la souplesse typique de la structure de réseau accroît chez les différents sujets la capacité de s'adapter au changement et de développer une intelligence collective où passé, présent et futur s'intègrent dans un modèle cohérent et stable, mais pas statique, du monde. La confrontation et les activités communes d'institutions, d'associations et de groupes d'un réseau peuvent faire émerger de nouvelles situations où la créativité du système est en mesure d'établir, et ensuite de maintenir, une sorte d'équilibre dynamique. En effet, les réseaux sont un outil pour faire de la recherche, pour développer la pensée, pour échanger des méthodologies et ils permettent aux systèmes complexes d'obtenir une réponse novatrice toutes les fois que surviennent des changements, comme cela se produit par exemple dans le contexte écologique, social et politique actuel, caractérisé par des situations critiques qui requièrent justement ces réponses. 
11 Le partenariat que le réseau implique et favorise permet de satisfaire des exigences d'ordre épistémologique, éthique et stratégique :

- d'ordre épistémologique parce que «le partenariat peut mettre à profit la diversité de ces regards sur le monde et de ces pratiques pour la construction d'un savoir plus riche, plus pertinent, plus crédible, plus utile, concernant l'environnement, et plus spécifiquement notre relation à l'environnement » et c'est le lieu d'un «dialogue de savoirs de type scientifique comme de types expérientiel, traditionnel, de sens commun, etc.» (Sauvé, 2001-2002);

- d'ordre éthique parce qu' « il incite à la prise en charge collective de cet objet partagé qu'est l'environnement. Il n'apparaît pas légitime (ni cohérent avec une vision systémique) de s'occuper individuellement de «son petit bout d'environnement». L'environnement est un objet politique («politique : qui concerne les choses publiques ») : les décisions doivent être partagées et les projets, collectivement conçus et assumés » (ibid.);

- d'ordre stratégique, parce que «le changement, qu'il soit environnemental, social ou éducationnel (ou les trois à la fois dans le cas de l'ERE) est a priori difficile à instaurer : il nécessite la concertation, la mise en commun de ressources et de compétences diverses » (ibid.).

1. L'institution éducationnelle profondément insérée dans le contexte social et engagée dans un dense réseau de relations et de partenariats est en syntonie avec l'idée contemporaine d'une société éducationnelle, d'une "communauté éducationnelle ( ou, selon l'échelle, " ville éducationnelle », " région éducationnelle ) qui englobe tous les facteurs politiques, sociaux, psychologiques, culturels, éducationnels et environnementaux et qui, en tant que telle, est aussi une «communauté écologique» (Capra, 2005). La participation des établissements scolaires et des universités à l'Agenda 21 local peut constituer un bon exemple d'engagement et de partenariat visant à l'éco-développement du territoire.

2. L'étroite union entre pensée et action, entre principes et pratiques réelles d'une ERE correctement instaurée satisfait les acquisitions de la pédagogie quant à la construction de la connaissance et la multiplicité des intelligences. L'efficacité didactique et éducatrice (visà-vis des capacités des élèves et de leur sens moral) des méthodes actives est largement démontrée. Grâce aux méthodes de recherche-action, à l'attention réservée à l'implication émotive, à l'utilisation d'un large éventail de techniques et d'outils didactiques, au rôle joué par l'opérationnalité et par la présence de situations réelles, au respect pour les différences, l'éducation va au-delà, par exemple, de la dichotomie entre l'esprit et le corps. Elle fait appel et met en cause des connaissances, des habiletés et des compétences diverses (théoriques, relationnelles, organisationnelles, etc.) et laisse libre champ aux différentes formes d'intelligence.

3. La dernière raison, mais pas pour autant la moins importante, concerne la contribution à la réduction de l'empreinte écologique de l'humanité sur la planète fournie par l'engagement pour la gestion cohérente d'un établissement scolaire ou d'un complexe universitaire où vivent des milliers d'étudiants et où se trouvent souvent des résidences, des cantines, des complexes sportifs, des aires de détente, des espaces verts.

\section{Écoles et universités « vertes »}

12 En effet, l'expérience nous enseigne que les écoles et les universités contribuent à l'entropie et qu'elles doivent donc obtenir des économies d'énergie, et de matière qui se traduisent par de moindres émissions de gaz à effet de serre, par une moindre exploitation de ressources naturelles, mais aussi par des économies d'argent pouvant 
être réinvesti dans de nouvelles améliorations environnementales et dans des services pour les étudiants.

13 Il ne faut pas oublier, entre autres, que le secteur de l'instruction et de la formation est aussi un sujet économique capable d'influencer le marché : les approvisionnements «verts» d'écoles ou, mieux encore, de groupements d'achats entre écoles et entre universités sont en mesure de stimuler l'offre de produits écologiques, de récompenser, par exemple, ceux qui ont une marque écologique, d'amorcer des dynamiques positives dans la production de biens et de services, de provoquer une réduction des prix des fournitures «durables». Ce secteur peut donc jouer un rôle très important non seulement d'exemple, mais de véritable "ouvre-piste», et favoriser ainsi une transformation à une échelle beaucoup plus vaste des styles de production et de consommation.

La nourriture représente un thème négligé, mais très intéressant: il faudra faire attention aux aliments servis dans les cafétérias internes et dans les cantines, car ils devront être de saison et biorégionaux (exception faite pour les produits effectivement nécessaires et non disponibles localement, qui dans ce cas devront être trouvés, par exemple, dans les circuits du commerce équitable et solidaire), essentiellement organiques, conditionnés de manière à obtenir une diminution des emballages, basés sur des menus qui encouragent (sans toutefois l'imposer) le régime végétarien, produire du compost avec la partie humide des déchets (restes de la restauration, mais aussi tonte des espaces verts et des terrains de sport), utiliser aussi les moments de fête comme occasion de pratique cohérente de la durabilité (par exemple en évitant les récipients jetables, en faisant attention au choix des aliments et des boissons, etc.).

Dans les achats en général, également pour les biens et les services non alimentaires, il faut préférer les fournisseurs locaux (cela sert à diminuer l'impact dû aux transports), assurer une diminution des emballages, réserver une attention particulière à l'élimination correcte des déchets, privilégier les équipements technologiques à faible consommation et les produits à marque écologique.

16 Le chapitre des transports concerne des mesures aisément envisageables comme l'encouragement à utiliser le vélo (création de pistes cyclables, installation de râteliers à vélos, conventions pour l'achat des bicyclettes, etc.), la marche à pied, l'emploi des transports en commun.

La qualité d'un environnement éducatif (tout comme, du reste, celle des autres lieux où se déroulent les activités humaines) est influencée par des paramètres physicochimiques, organisationnels, architecturaux, etc., dépendant en partie des caractéristiques du bâtiment ou des bâtiments qui le composent, en partie de facteurs externes.

Dans les restructurations et dans les nouvelles constructions, tout comme pour les aménagements intérieurs, il faudra réserver une attention particulière à la bioarchitecture par l'emploi de matériaux ayant un cycle de vie écologique, en réservant une attention particulière à l'isolation, et en appliquant les différentes solutions offertes par la bioclimatique pour protéger du froid et de la chaleur tout en économisant de l'énergie. L'attention pour l'environnement dans le bâtiment et dans l'ameublement se manifeste également par de nombreux autres aspects, par exemple en évitant les peintures et les colles nocives ou les bois provenant de forêts primaires, en utilisant des installations à haute efficacité, en prévoyant des systèmes pour 
l'économie et la récupération de l'eau, en recourant à des énergies renouvelables (grâce aux panneaux solaires), en recyclant les matériaux de démolition, etc.

La qualité de la vie dans une structure éducative et sa durabilité dépendent aussi de ce qui est autour : par exemple des cours qui peuvent être transformées en espaces verts (sans oublier les abris pour les animaux), des espaces à requalifier dans le sens environnemental et d'autres facteurs externes. Une rue à grande circulation ou une usine produira de la pollution atmosphérique et sonore, une ligne électrique pourra émettre des champs magnétiques, le quartier pourra être marqué par la violence et la dégradation socio-environnementale, etc. Il faudra donc s'occuper de cela aussi.

Parmi les paramètres strictement environnementaux, généralement fixés par la réglementation pour la protection de la santé, de la sécurité et du confort, il faut citer la disponibilité d'espace par personne, la température, l'humidité, l'éclairage, la qualité de l'air, la situation sonore, les mesures antisismiques ou anti-incendie, toutes les prescriptions visant à prévenir les accidents de différents types aussi bien dans les salles de classe que dans les laboratoires (décharges électriques, chutes, ruptures de vitres, etc.), les sanitaires, etc., et l'état d'entretien de tout ceci.

21 Un aspect important de l'école "écologique » est sans aucun doute qu'elle doit être sûre pour ceux qui l'« habitent » et qu'elle doit enseigner la sécurité : en effet, l'école est aussi un maillon important d'un système éducationnel de protection contre les catastrophes.

La préparation d'écoles et d'autres structures éducatives se traduit par la capacité de résister à n'importe quel danger, par des enseignants qui savent quoi faire en cas de catastrophe, par des élèves conscients des risques et capables d'en reconnaître les signaux, par des parents qui savent pouvoir compter, en cas de catastrophe, sur l'école comme lieu sûr et avec la présence d'un personnel préparé, par des plans adaptés aux menaces possibles, par des plans d'urgence au niveau de la communauté locale coordonnés avec les plans scolaires, par l'introduction du thème des catastrophes dans le programme d'études, par des exercices annuels de simulation avec la participation des enseignants, des élèves et des parents. Si la catastrophe se produit, une fois que l'urgence est passée, l'école doit rapidement rouvrir ses portes et garantir la continuité du service éducatif (UNESCO et PNUE, 2004).

\section{Écologie des relations interpersonnelles et de la communication}

Parmi les paramètres organisationnels et relationnels, nous pouvons citer la quantité, la qualité, l'efficience, le niveau d'utilisation des équipements technico-scientifiques, les laboratoires, les bibliothèques et les autres outils didactiques, la discipline, la communication interne enseignants-enseignants, enseignants-apprenants et apprenants-apprenants, la conception du processus d'enseignement/apprentissage, le climat humain, le caractère démocratique et la transparence de la gestion, le professionnalisme et l'enthousiasme des enseignants, la motivation des étudiants, le contexte social, le rapport avec la collectivité, l'ouverture au territoire, etc.

Le chapitre communication aborde aussi le thème des technologies de l'information et de la communication (TIC). Les technologies sont très utiles et se prêtent à de très nombreux usages, pour être constamment mises à jour, pour travailler en réseau avec 
d'autres, pour accéder à des banques de données et à des ressources en ligne, mais elles doivent aussi être utilisées à bon escient et il faut en éviter les excès. Les TIC peuvent rendre le rapport enseignant- apprenant plus interactif, elles aident à réduire l'empreinte écologique des activités (par exemple en réduisant l'emploi de papier ou les déplacements), elles reflètent la réalité d'un monde où les TIC ont une présence croissante. Cependant, il ne faut pas oublier que la disponibilité et l'accès aux technologies ou la capacité de les utiliser et de les maitriser ne sont pas répartis de manière équitable entre les peuples et entre les classes sociales. Par ailleurs, comme dans les autres milieux d'apprentissage, elles doivent tenir compte des différents niveaux de complexité organisationnelle, d'échelle temporelle, d'échelles et de relations spatiales.

Il ne faut donc pas se limiter à assurer l'accès aux nouvelles technologies. Par ailleurs, toute activité devra être considérée comme une composante du vaste environnement d'apprentissage dans lequel nous vivons. Il faudra aussi faire en sorte que les environnements virtuels aient les mêmes caractéristiques que les environnements physiques : les personnes doivent pouvoir interagir entre elles et avec toutes les autres composantes de la société humaine et civile.

\section{Engagement direct des étudiants}

d'en faire une occasion de travail commun des enseignants et des apprenants, en proposant aux étudiants des activités multidisciplinaires, avec des applications pratiques des notions « apprises sur le tas » et un espace pour l'initiative directe de la part des jeunes.

À ce propos, il faudra aussi favoriser l'activisme des étudiants. Dans grand nombre de pays des cinq continents, ce sont les étudiants qui prennent directement en main l'engagement envers la durabilité d'écoles et d'universités. Ils ont ainsi constitué des groupes de travail et des associations ad hoc qui prennent différents noms (en français, on trouve par exemple: «clubs environnement », «clubs verts», «clubs écologie», etc., en anglais les équivalents «ecoclubs », « environmental clubs», " green clubs ", «ecology clubs», «envi-clubs», etc.; dans certains cas, le club est dédié à un personnage ou son nom est inspiré d'un aspect de l'environnement ou d'un slogan). L'objectif des clubs est de promouvoir la sensibilité vis-à-vis de l'environnement et - en contribuant à réduire l'impact sur l'environnement en tant qu'individu, institution et collectivité - d'accroître la durabilité de leur institution (école ou campus) et indirectement de leur communauté. Il existe des clubs de ce genre dans des centaines d'écoles, d'universités et d'instituts d'enseignement supérieur du monde entier, de la France à l'Algérie ou au Liban, de la Chine à la Jordanie, de la Suède à la Turquie et dans 
tout le monde anglo-saxon (Australie, Canada, Nouvelle-Zélande, Royaume-Uni, ÉtatsUnis).

\section{Une éducation « écologique »} (2001) - au sein d'un paradigme éducationnel occidental mécaniste et réductionniste, de plus en plus managérial et orienté vers un savoir-marchandise. Le paradigme éducationnel doit donc être revu dans le sens «humaniste, démocratique et écologique ", à la lumière d'une "écologie de l'éducation" qui perçoit le système éducationnel, son éthos, son programme d'études, sa communauté comme un tout interrelié (Sterling, 2001, p. 14). Il s'agit, affirme Sterling, de passer d'un apprentissage transmissif à transformatif, c'est-à-dire critique et créatif, attentif aux différentes formes de l'intelligence humaine comme aux différences individuelles et de groupe, orienté vers l'avenir, visant à développer la compréhension de la complexité croissante et de l'interdépendance du monde contemporain. C'est tout le système qu'il faut changer, en basant les processus d'enseignement/apprentissage sur la curiosité, sur l'imagination, sur l'enthousiasme, sur l'esprit de changement et en devenant capable de construire une vraie société cognitive, capable à même de se comprendre et de se réorienter.

31 L'éducation écologique (ou "éducation durable ») signifie donc agir au niveau des valeurs de base, du programme d'études, de l'évaluation et des contrôles, de l'organisation, de la gestion, de la construction de bâtiments scolaires, du rapport avec la communauté locale, des méthodologies, de l'attention réservée aux élèves, des styles d'enseignement et d'apprentissage, du concept même d'apprentissage (Salomone, 2005).

Bref, l'ERE demande à l'institution éducationnelle aussi bien d'introduire l'écologie dans l'éducation (c'est-à-dire dans le programme d'études) que de réaliser une écologie de l'éducation (en considérant en quelque sorte toute école comme un écosystème) et une écologie du système. À savoir, il s'agit donc d'introduire une écologie des relations de l'institution avec l'ensemble de la réalité sociale et de toute l'offre éducationnelle présente dans les divers contextes territoriaux, à différents niveaux de complexité organisationnelle, d'échelle temporelle, d'échelles et de relations spatiales. 


\section{BIBLIOGRAPHIE}

Amerio, P. et Croce, M. (2000). Reti sociali. In Amerio, P. (dir.), Psicologia di comunità (p. 331-363). Bologna : Il Mulino.

Becattini, G. et Sforzi, F. (2002). Lezioni sullo sviluppo locale. Torino : Rosenberg et Sellier.

Bocchi, G. et Ceruti, M. (2004). Educazione e globalizzazione. Milano : Raffaello Cortina Editore.

Capra, F. (2005). Ecological Literacy: The Challenge for Education in the 21st Century. Communication présentée au « 3rd World Environmental Education Congress », 2-6 octobre 2005, Torino, Italie.

Coluccia, P. (2001), La Banca del Tempo. Un'azione di reciprocità e di solidarietà. Torino : Bollati Boringhieri.

Coluccia, P. (2002). La cultura della reciprocità. I sistemi di scambio locale non monetari. Casalecchio (BO) : Arianna Editrice.

Dubet, F. (2002). Le déclin de l'institution. Paris : Seuil.

Grasselli, P. (2003). La dimensione relazionale nello sviluppo locale. Communication présentée au colloque de la Fuci « Le nuove dimensioni del rapporto tra etica ed economia », 6 novembre 2003, Terni.

Orr, D.W. (1992). Ecological Literacy. Education and the Transition to a Postmodern World. Albany : State University of New York.

Orr, D.W. (1994). Earth in Mind. On Education, Environment, and the Human prospect. Washington (D.C.) - Covelo, California : Island Press.

Piselli, F. (2001). Reti. L'analisi di network nelle scienze sociali. Roma : Donzelli Editore.

Prigogine, I. (1999). The Die is not cast. In Mayor, F. et Droit, R.-P. (dir.), Letters to future generations (p. 133-140). Paris : UNESCO.

Sacco, P.L. et Zamagni, S. (2002). Complessità relazionale e comportamento economico.Bologna : Il Mulino.

Salomone, M. (2005). Strategie educative per la sostenibilità. L'educazione ambientale nel XXI secolo. Bergamo : CELSB.

Sauvé, L. (2001-2002). Le partenariat en éducation relative à l'environnement : pertinence et défis. Éducation relative à l'environnement : Regards - Recherches - Réflexions, 3, 321-336. Sterling S. (2001). Sustainable Education: Re-Visioning Learning and change. Devon : Green Book.

UNESCO et PNUE, Awareness and Preparedness for Emergencies at the Local Level (APELL) (2004). APELL for Schools Educational Buildings : A community-based approach for school safety and education for disaster reduction. Paris : UNESCO.

WEEC (2005). Principes directeurs du Congrès mondial de Turin pour une éducation relative à l'environnement de grande envergure, fondée sur des bases éthiques et épistémologiques solides. Torino : WEEC. 


\section{RÉSUMÉS}

$\mathrm{Au}$ nom d'un principe de cohérence entre finalités, contenus et organisation du système éducationnel, il faut repenser et réorganiser du point de vue écologique non seulement le programme d'études, mais aussi les méthodologies, le rapport enseignant-apprenant, les milieux d'apprentissage considérés aussi bien au sens strict (espaces physiques où se déroulent des activités d'apprentissage structurées) qu'au sens large du terme, comme "communauté éducationnelle » qui comprend tous les facteurs politiques, sociaux, psychologiques, culturels, éducationnels et environnementaux. En effet, l'éducation relative à l'environnement peut représenter un moteur de changement surtout si elle devient le laboratoire et l'exemple concret d'une réorientation culturelle et socioéconomique, à travers l'écologie des bâtiments, la réduction des émissions, la pratique de styles de vie et de comportements écologiques, l'écologie de l'organisation des temps, l'écologie de la communication interne, l'écologie de l'utilisation des structures, l'écologie des réseaux et des partenariats. Bref, il s'agit de réaliser une écologie dans l'éducation, une écologie de l'éducation et une écologie du système.

Many features such as methodologies, teacher-learner relationship, learning spaces (both in terms of physical space and of an "educational community", which includes political, social, cultural, educational and environmental factors) are to be re-thought and re- oriented, in order to obtain a coherent link between the aims, contents and organization of the whole educational system. Environmental education (EE) can contribute to the transformation of some cultural and socio-economical settings, through the greening of buildings, emissions' reduction, ecological life-style promotion and reconsideration of time spending, internal communication and facility use. Everyday our definitive duty is to perform ecology in education, ecology of education and ecology of the system itself.

\section{AUTEUR}

\section{MARIO SALOMONE}

Professeur d'ERE à l'Université de Bergame (Italie), il est directeur et fondateur du mensuel italien de l'ERE Eco l'educazione sostenibile. Il a organisé le 3e WEEC - World Environmental Education Congress(octobre 2005) - et il a accompagné plusieurs projets éducatifs aux niveaux national et international. Il est aussi engagé dans le Secrétariat permanent des congrès mondiaux de l'ERE. Ses ouvrages récents sontMondo, mondo dentro. Un percorso nella complessità ambientale (2004) et Strategie educative per la sostenibilità. L'educazione ambientale nel XXI secolo (2005). 